\title{
A EXPANSÃO E AS POSSIBILIDADES DE DEMO- CRATIZAÇÃO DO ENSINO SUPERIOR NO BRASIL
}

\author{
Antonio Augusto Pereira Prates* \\ Maria Ligia de Oliveira Barbosa * *
}

\begin{abstract}
Diante da expansão e diversificação recentes do sistema de ensino superior brasileiro, este artigo discute as possíveis tendências à sua modernização e democratização. O artigo busca desenvolver questões fundamentalmente associadas aos valores socialmente atribuídos aos diplomas do ensino superior, principalmente na relação entre o domínio quase absoluto de uma visão patrimonialista sobre o ensino superior e o esgarçamento dos conteúdos científicos e (ou) pedagógicos associados aos diversos diplomas. O patrimonialismo aparece na preferência pelo bacharelado, em detrimento dos diplomas de licenciatura e tecnológicos, nas vocações socialmente marcadas, nos horários e turnos dos diferentes cursos e modalidades, no desinteresse pelas áreas técnicas e na pedagogia do cultivo. Mais ainda, o patrimonialismo aparece na dialética entre saberes e a força social dos diplomas. A possível instalação de um credencialismo radical no Brasil permite analisar a relação entre o domínio patrimonial e a cultura profissional, indicando os limites da democratização do sistema de ensino superior.
\end{abstract}

Palavras-chave: Ensino Superior. Credenciais. Cultura profissional. Patrimonialismo.

Por longos anos, o Sistema de Ensino Superior (SES) no Brasil, como em todo o mundo, foi claramente um espaço para socialização de uma elite mais preparada, distinta - pelo seu capital cultural certificado - dos mandantes e mandatários espalhados por todo o país. Se as disputas entre os setores da elite permitem a Simon Schwartzman (1987) opor os saberes modernos aos tradicionais como recursos utilizados nas disputas por poder, os estudos de Octavio Dulci $(1983 ; 2002)$ sobre os pactos das elites mineiras indicam que nem tão distintas são as faces diferentes desse grupo. Já na primeira metade do século XX, doutores e coronéis se davam muito bem na organização e regulação da vida social. "Os donos do poder" conhecem as regras do jogo social e as estratégias de convivência dentro desses quadros.

No entanto, nas últimas décadas, como mostrou Carlos Benedito Martins (2006), for-

* Universidade Federal de Minas Gerais, Faculdade de Filosofia e Ciências Humanas, Departamento de Sociologia e Antropologia.

Av. Antonio Carlos, 6627. Pampulha. Cep: 31270-901. Belo Horizonte - Minas Gerais - Brasil aaprates@oi.com.br

** Universidade Federal do Rio de Janeiro, Instituto de Filosofia e Ciências Sociais, Departamento de Sociologia. Largo de São Francisco 1. Centro. Cep: 20051070. Rio de Janeiro - Rio de Janeiro - Brasil mligiabarbosa@gmail.com mou-se, no país, um complexo campo acadêmico, desenvolvendo-se um sistema de ensino superior relativamente ampliado e inclusivo. A matrícula desse sistema, em todos os seus níveis, passa de 1 milhão de estudantes em 1998 para, aproximadamente, 7,5 milhões em 2012, segundo o Censo do Ensino Superior do MEC. Tanto por essa expansão significativa da matrícula - que, mesmo assim, ainda nos deixa bastante distante dos níveis de participação atingidos em países desenvolvidos: 42\% dos jovens entre 18 e 24 anos nos Estados Unidos em 2011 (dados do National Center for Education Statisitcs) contra os nossos atuais $15,10 \%$ de matrícula líquida em 2012 - quanto pela implementação de diversas políticas institucionais, principalmente as cotas, verifica-se uma entrada importante de estudantes negros e de oriundos das classes populares.

Martins (2006 p. 1002) destaca o caráter global desses processos:

Uma das tendências centrais do ensino superior contemporâneo, em escala internacional, diz respeito à ampliação do seu acesso, fenômeno que se iniciou a partir da segunda metade do século XX. Um conjunto de fatores tem contribuído para esse 
processo, tais como a valorização do conhecimento técnico e científico como um dos ingredientes centrais das sociedades modernas, pressões por direitos sociais, aspirações de mobilidade social por meio do sistema educacional, por parte dos estudantes e de suas famílias, necessidade da aquisição de competências técnicas para enfrentar um mercado de trabalho cada vez mais instável e seletivo, transformações no conteúdo das profissões, trazendo de volta para os bancos escolares uma população adulta e já integrada em atividades profissionais etc.

Um ponto importante a anotar aqui seria o fato de que o ensino superior deixa de ser, segundo Martins, voltado para sua tradicional e restrita clientela de elite e passa a incorporar parcelas de alguns grupos que antes não passavam por essas instituições. O grande número de alunos que são os primeiros de suas famílias a chegarem a esse nível de ensino é uma evidência dessa incorporação.

O mesmo estudo destaca ainda as tendências de retração do setor público e de expansão do setor privado, assim como uma ampliação das funções das instituições do ensino superior. Fenômeno que acontece no mundo todo, esse crescimento foi acompanhado, no caso brasileiro, de um processo intenso de diferenciação institucional: ao lado das tradicionais universidades, surge uma infinidade de centros universitários e faculdades isoladas, bem como $\stackrel{2}{\stackrel{2}{~}}$ institutos de educação técnica ou tecnológica : em nível superior. Mais que isso, identificam$\sum_{\text {L }}^{\infty}$ se novas formas de diversificação técnico-funcional: novos temas, áreas recém delimitadas iे na divisão técnica do trabalho, especialidades

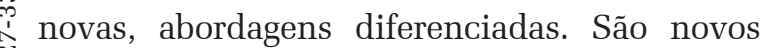
m

д quadro. E, obviamente, surgem novos diplomas

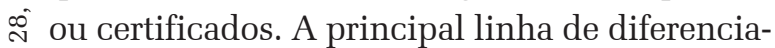
ção aparece nos três tipos de cursos possíveis. Permanece o bacharelado e especificam-se os cursos de licenciatura, que deixam de ser constituídos de meras disciplinas complementares aos bacharelados e se tornam cursos independentes. Também se desenvolve a alternativa de formação superior tecnológica.
Temos, então, um modelo de SES moderno e democrático? Essa seria uma questão essencial, como já foi sugerido no estudo mencionado de Carlos Benedito Martins e pelo texto de François Dubet, também neste dossiê. De forma ampla, pode-se dizer que se trata de compreender a relação entre políticas que buscam melhorar o coeficiente de igualdade de chances educacionais entre pessoas de estratos e grupos distintos na sociedade, e os critérios de equidade que permeiam essas políticas. Em processos de expansão do sistema de ensino ancorados, inclusive, em diversas políticas públicas, como foi o caso brasileiro, essa seria uma reflexão da maior importância para qualificar de forma lúcida e tornar menos ingênuas essas próprias políticas e a nossa compreensão das possibilidades efetivas de democratização desse sistema. Essa discussão enquadra-se perfeitamente no contexto brasileiro atual. Desde as políticas de expansão do sistema público, passando pelas políticas de cotas e de bolsas estudantis, como muito bem mostra Dubet, é totalmente pertinente a discussão da equidade para evitarmos simplificações sociológicas que possam comprometer os resultados dessas políticas.

Mesmo fora do ambiente acadêmico, surgem questões importantes sobre um possível sucateamento dos diplomas (o retorno da inflação de diplomas, tão combatida por Bourdieu?), ou sobre a natureza excessivamente acadêmica (currículo muito extenso e pouco prático, beirando a inutilidade no mercado de trabalho). Não faltam também as queixas sobre a incapacidade de formação da mão de obra qualificada necessária num mundo do trabalho mais complexo e moderno. Os debates recentes sobre a falta de mão de obra qualificada em algumas áreas, particularmente as engenharias, têm evidenciado algumas dessas mazelas. ${ }^{1}$

Nesse quadro, reforça-se a questão sobre os sentidos sociais atribuídos ao sistema de

${ }^{1}$ A coletânea publicada pela Rede de Pesquisa Formação e Mercado de Trabalho, ABDI/IPEA, expressa bem esses debates. Disponível em < http://redebrasileirademea.ning. com/profiles/blogs/rede-de-pesquisa-forma-o-e-mercadode-trabalho-colet-nea-de $>$. 
ensino superior em suas novas condições, que devem ser ainda melhor caracterizadas a partir de abordagens sociológicas. Nosso artigo busca desenvolver questões fundamentalmente associadas aos valores socialmente atribuídos aos diplomas do ensino superior e que se expressam de forma contundente na relação entre o domínio quase absoluto de uma visão patrimonialista sobre esse sistema de ensino e o esgarçamento dos conteúdos científicos e (ou) pedagógicos associados aos diversos diplomas, mas também na relação entre os formatos institucionais do ensino superior e as posições ou ocupações no mercado de trabalho. Dessa forma, tentamos avançar na análise ${ }^{2}$ da expansão do ensino superior deixando de lado algumas perspectivas mais descritivas e buscando construir uma abordagem propriamente analítica dos processos sociais constitutivos desse crescimento.

\section{DOMÍNIO DE UMA VISÃO PATRIMO- NIALISTA NO ENSINO SUPERIOR}

São suficientemente significativos os estudos sobre a força do patrimonialismo na sociedade brasileira, mostrando seus efeitos sobre as práticas políticas e administrativas que até hoje perduram no seu interior. Neste artigo, estenderemos um pouco o conceito de patrimonialismo para o espaço do ensino superior. Obviamente, consideramos que as análises conhecidas e reconhecidas de Sérgio Buarque de Holanda e Raymundo Faoro, assim como aquelas de Schwartzman, permitem esse tipo de extensão.

Se, para Weber, o Patrimonialismo expressa uma forma tradicional de exercício da dominação, na

[...] experiência brasileira, as análises de Faoro sobre o papel histórico da tradição patrimonial-buro-

${ }^{2}$ Ver, a esse respeito, o livro organizado por Margaret Archer (1982), em particular a introdução. Nesse trabalho, a autora inglesa busca formular conceitos que possam efetivamente explicar os processos sociais envolvidos na expansão e desenvolvimento dos diversos sistemas de ensino superior em cada país. crática portuguesa e seus prolongamentos no país abrem caminhos importantes de pesquisa, em termos de suas transformações, choques e conflitos com outras tendências também presentes, como as do capitalismo moderno e as derivadas da política de massas (Schwartzman, 2013, p. 210).

Acrescentaríamos, utilizando uma expressão do próprio Schwartzman (1987), a tendência de implantação dos "conhecimentos modernos” na universidade brasileira.

Uma das características fundamentais da dominação patrimonial é o caráter personalista e casuístico das relações sociais, que se oporia ao ordenamento moderno dessas mesmas relações, onde prepondera uma racionalidade universalista e racional-legal. Nas formas tradicionais, prevalece a vontade do senhor. Em sociedades modernas, dominariam as regras (Campante, 2003), que tenderiam a ter validade o mais universal possível.

É interessante notar que, entre os poucos estudos na área de educação que recorrem ao conceito de patrimonialismo, essa forma social aparece normalmente como entrave aos processos de democratização em andamento (Mendonça, 2000, por exemplo). Reforçando essa ideia de que o patrimonialismo entrava a instalação de procedimentos democráticos na gestão do sistema público de educação, buscamos, aqui, caracterizar e enfatizar alguns traços do patrimonialismo que seriam essenciais para o próprio funcionamento do ensino superior brasileiro.

\section{OS TRAÇOS PATRIMONIALISTAS NO SISTEMA DE ENSINO SUPERIOR}

\section{A preferência pelo bacharelado}

Traçar as linhas do que seriam as características patrimonialistas do nosso SES significa delimitar aqueles atos e valores praticados regularmente e distintamente nas instituições desse sistema. As formas dominantes dos processos educativos na sociedade brasileira, as concep- 
ções mais fortes que definem o seu funcionamento acabam se expressando nas práticas cotidianas dos atores do SES. É fundamental entender quais são essas regularidades no funcionamento do SES e quais os seus efeitos sobre as trajetórias sociais distintas dos indivíduos que passam ou tentam passar por esse sistema.

O primeiro desses traços seria a preferência ampla - tanto do ponto de vista do número de cursos quanto daquele de alunos - pela formação nos bacharelados. Dos quase 30.000 cursos que funcionavam em 2010, mais da metade $(55,6 \%)$ eram bacharelados.

Tabela 1 - Número de cursos segundo o grau acadêmico

\begin{tabular}{llcr}
\hline Grau & & N. Cursos & \% \\
\hline Validos & Bacharelado & 16.586 & 55,6 \\
& Licenciaturas & 7.922 & 26,6 \\
& Tecnológicos & 4.999 & 16,8 \\
& Total & 29.507 & 98,9 \\
Missing & System & 328 & 1,1 \\
\hline Total & & 29.835 & 100,0 \\
\hline
\end{tabular}

Fonte: Censo Educação Superior/INEP/ 2010

De outro lado, também se verifica que a evolução do número de matrículas por grau acadêmico, entre 2001 e 2010, reafirma o predomínio dos bacharelados, apesar da entrada dos tecnólogos como uma alternativa crescente. Para que se possa ter uma ideia da amplitude desses números, em 2001

$\stackrel{5}{5}$ foram 3.036.113 matrículas e, . em 2010, chegou-se a um total

de 6.379.298. As matrículas nos cursos tecnológicos passaram de 2,30\% em 2001 para 12,25\% do total em 2010. Já a matrícula nos bacharelados + oscila entre $67,08 \%$ e $66,26 \%$ a no mesmo período. Conside-

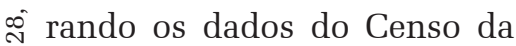

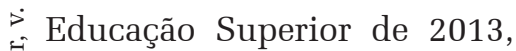
essa situação se acentua ainda um pouco mais, pois os bachai relados assumem $67,50 \%$ das matrículas e as licenciaturas

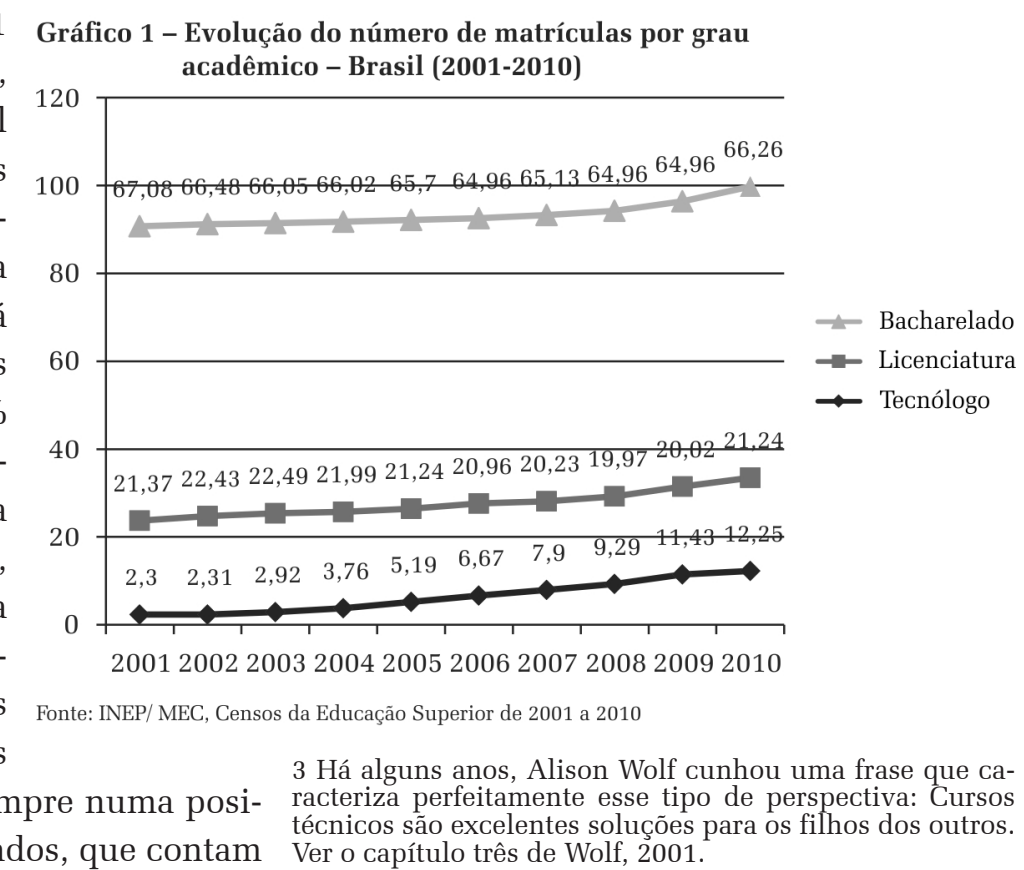

com mais de dois terços das preferências.

As possíveis razões para essas tendências da abertura de cursos, por um lado, e das matrículas, por outro, são variadas, pois é possível distinguir critérios e racionalidades distintas. As decisões de abrir novos cursos, quais cursos e o tipo de grau a ser conferido a eles são orientadas por lógicas diferentes daquela do indivíduo que procura se qualificar. A decisão desse último tanto pode ser orientada pelo desejo de realização pessoal quanto por critérios instrumentais que visam a obter o maior rendimento possível do seu investimento na escolarização.

\section{Preferências socialmente marcadas e horá- rias dos cursos}

Seria importante destacar também que essas preferências pelo tipo de grau são bastante marcadas do ponto de vista social: cursos de licenciatura ou tecnológicos são - em alguns casos, com fortes indicações de preconceitos por parte tanto de famílias quanto de agentes do próprio sistema de ensino ${ }^{3}$ - "preferidos" pelos alunos de origem mais modesta. Já o estudo de Braga et al (2001) indicava essa tendência para a Universidade Federal de Mi- 
nas Geral - UFMG, com um claro aumento da demanda para os cursos menos prestigiados e de licenciatura para alunos de grupos sociais menos afluentes. Já para os alunos das classes médias educadas, a escolha recaía principalmente sobre os cursos muito prestigiados e com um número muito elevado de alunos disputando cada vaga. Como já havia sido indicada por Paul e Valle Silva (1998), a existência de cursos noturnos é um fator decisivo para garantir a entrada e a permanência de estudantes de origem social mais modesta. Também no artigo de Braga et al (2001) e naquele de Zago, 2006, os horários de funcionamento dos cursos superiores permitem aumentar efetivamente as oportunidades de acesso e permanência dos alunos de classes populares. A esse respeito, as informações sobre os cursos noturnos e diurnos, segundo a categoria administrativa da instituição, permitem avaliar o quanto se poderia esperar, nesse item específico, de abertura efetiva do SES às classes populares.

Percebe-se nitidamente, nesses dados, que as universidades de elite, principalmente as públicas federais, têm as menores proporções de matrículas em cursos noturnos. Desse ponto de vista pelo menos, parecem ser as instituições privadas aquelas que oferecem maiores oportunidades para a permanência dos estudantes mais pobres. Obviamente, esse não é o único fator.

Gráfico 2 - Matrícula (\%) por turno, segundo categoria administrativa

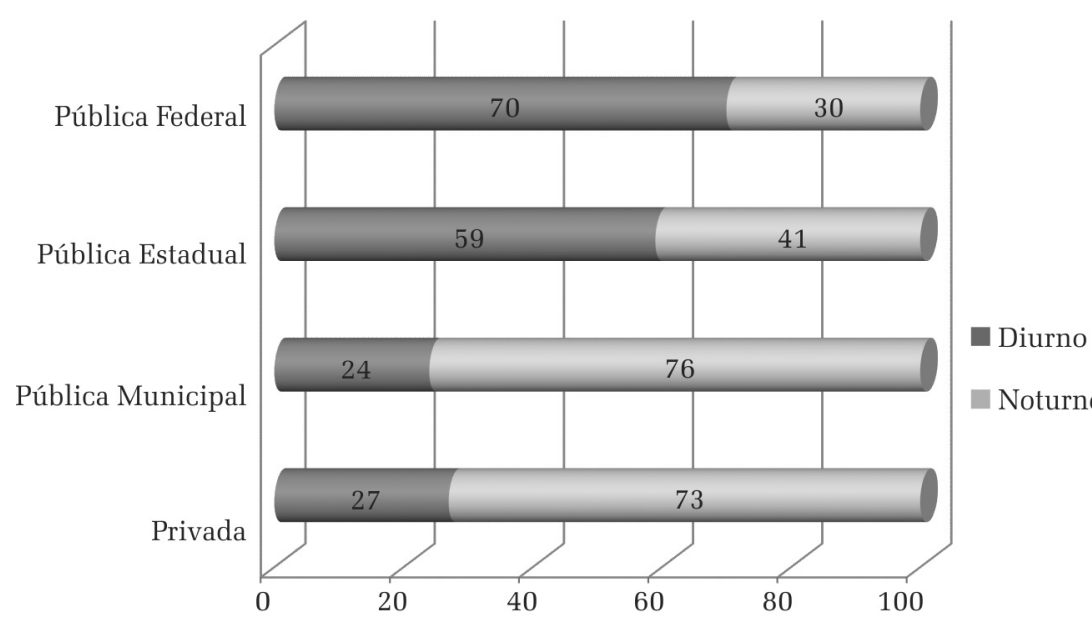

Fonte: MEC/INEP, Censo da Educação Superior 2013
Mas, na medida em que se somam outras características do modo de funcionamento do SES no Brasil, a distribuição dos alunos e cursos pelos diferentes turnos pode indicar a existência de formas ou mecanismos que tornam menos fácil e menos adequada (ao tipo de vida desses alunos) a permanência de estudantes das classes populares na universidade pública. É sempre bom lembrar que estratégias sociais, como mostram estudos da escolha racional e de linha bourdieusiana, não são plenamente conscientes, seja pela ausência de informações completas, seja pela marca da posição de classe na constituição do habitus, que tende a naturalizar uma determinada perspectiva específica como sendo universal. Desse segundo ponto de vista, o fato de entrar para uma universidade de elite já é um ganho (certamente é), e o indivíduo que consegue esse feito não se aprofunda na relegação que é feita no interior dessa mesma instituição (os famosos excluídos do interior, de Pierre Bourdieu), tanto pelos horários quanto pelo desprestígio social das carreiras efetivamente abertas aos menos afluentes.

\section{Desinteresse pelas áreas técnicas}

Esse domínio do bacharelado não poderia ser tratado, em si mesmo, como um indicador da presença de uma visão patrimonial no interior do sistema de ensino superior. No entanto, além da massiva preferência pelo título de bacharel, o fato de que a opção pelas licenciaturas e pelos cursos tecnológicos seja feita pelos estudantes originários das camadas menos afluentes da população e que agora têm acesso ao ensino terciário reforçaria o argumento patrimonialista. Ainda que as desigualdades entre 
os percursos escolares sejam uma característica de quase todos os sistemas de ensino (Barbosa, 2012, Prates; Faleiro, 2014; Shavit, 1993, 1998; Brunello, 2007), sempre com maior prestígio e recompensa econômica para o ensino acadêmico e para o bacharelado, o caso brasileiro parece agregar dimensões quase senhoriais aos diplomas. Elas se traduzem, inicialmente, numa desvalorização das carreiras e dos trabalhos técnicos - como mostram, de forma bastante clara, os debates sobre a falta de engenheiros (Gusso; Nascimento, 2011; Nascimento, 2014; Barbosa; Zuccarelli, 2014) e outros profissionais qualificados nessa área. É interessante notar a diminuta proporção de formandos em engenharias e ciências no Brasil (11,2\%), diante do gigantismo chinês (mais de 45\%) e também da robustez dos números da Organização para a Cooperação e Desenvolvimento Económico (OCDE) ou da União Europeia (acima de 20\%) (Gusso; Nascimento, 2011). Já na Fundação da Escola de Minas de Ouro Preto, em 1876, faculdade de engenharia em moldes modernos e arrojados, o Imperador D. Pedro II foi "obrigado" a transferir alunos do Rio de Janeiro para a instituição mineira, visando a garantir o seu funcionamento. Os filhos das elites burocráticas e agrícolas de Minas Gerais davam preferência aos cursos de Direito em São Paulo, Recife ou Coimbra. A en-

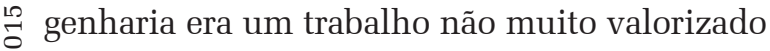
(Carvalho, 1976; Adorno, 1988).

Obviamente, esse tipo de dado apenas indica um padrão de valorização das diferentes ocupações, característico de cada sociedade. Ao longo dos anos, a procura pelas engenharias e por outras carreiras de perfil mais ‡ técnico ou científico é variável. No entanto, \& parece prevalecer, entre os filhos das elites 今 brasileiras, um conjunto de noções pouco afei-

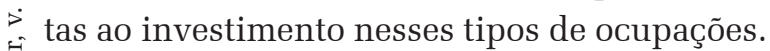

\section{A pedagogia do cultivo}

Outro traço patrimonialista seria a fortíssima presença do que se denomina a pedago- gia do cultivo. Esse termo já foi cunhado classicamente por Weber, que se referia a ela como sendo a forma educativa do patrimonialismo, o princípio da dominação tradicional. Análises do discurso dos pensadores da educação brasileira mostram a força dessa orientação, particularmente entre aqueles ocupantes de posições mais à esquerda no espectro político. Entre eles, a pedagogia do cultivo seria a orientação dominante, que se mostra particularmente enfática no desprezo sistemático pela formação técnica e tecnológica, vista como mero adestramento. $\mathrm{O}$ ideal, para qualquer tipo de atividade, inclusive trabalhos técnicos, seria a pessoa cultivada, que se distinguiria pela extensão da sua cultura geral. Assim, toma-se o homem culto do século XIX como o modelo do que deveria ser o indivíduo contemporâneo (Barbosa, 2010).

Esses importantes agentes da elaboração de políticas públicas, inclusive para o ensino superior, partilham essa visão do mundo, que se propõe a ignorar a divisão técnica do trabalho, inclusive no campo científico. Desse ponto de vista, apenas importa a divisão entre quem concebe ou dirige o trabalho e aqueles que o executam. Qualquer formação ou treinamento mais especializado é visto como um anátema que deve ser extirpado, pois denigriria o homem integral, tal como é entendido nessa perspectiva. São propostas formações enciclopédicas e superficiais em detrimento de abordagens tecnicamente fundamentadas e conteúdos cientificamente trabalhados.

Poder-se-ia traduzir a pedagogia do cultivo, na forma brasileira atual, pela preferência explícita por generalidades supostamente cultivadas à especialização competente (Barbosa, 2014).

\section{Dialética entre saberes e a força social dos diplomas}

Ainda como fator de caracterização do patrimonialismo no SES brasileiro, pode-se considerar a dialética entre o conteúdo dos saberes e dos conhecimentos e a força dos di- 
plomas. O domínio do título ou credencial parece separar-se do conteúdo técnico e mesmo de uma visão iluminista do mundo associada ao domínio do profissionalismo cientificista, e dos chamados "saberes modernos". É como se os títulos e diplomas fossem relevantes não pelo seu conteúdo em termos de conhecimentos aprendidos, mas pelo fato de oferecerem direitos e poderes sociais a seus portadores. Obviamente, não se trata de dizer que um advogado poderia substituir um cirurgião. No entanto, boa parte das formações tecnicamente distintas como física ou biologia acaba se abrindo para trabalhos não distinguíveis tecnicamente (Ojala, 2008). Caberia perguntar se a visão mencionada anteriormente - que prefere ignorar a divisão técnica do trabalho - produz efetivamente profissionais pouco distintos, que, mesmo assim, são absorvidos pelo mercado de trabalho, ou se esses profissionais indistintos apenas conseguem os espaços menos prestigiados desse mercado.

\section{Um credencialismo radical no Brasil?}

Particularmente nesse item, essa análise aproxima-se muito de uma perspectiva radicalmente credencialista que, do nosso ponto de vista, seria exagerada. Em tal perspectiva, como mostra Bills (2004), os diplomas teriam funções exclusivamente sociais e nenhum conteúdo técnico. Mesmo reconhecendo o valor posicional atribuído à educação em nossa sociedade, é possível ainda distinguir processos de formação profissional que geram identidades sociais diferenciadas e, inclusive, constituem-se em espaços para organização e ação coletiva (Barbosa, 2003). No entanto, mesmo assim, o nosso SES parece, por vezes, dar mais ênfase à credencial concedida que à formação, ao aprendizado e até mesmo a algum tipo de mérito que pudesse estar associado à passagem pela universidade. A valorização maior da credencial que do seu conteúdo pode ser percebida, por exemplo, em políticas aventadas para corrigir o problema da repetência (repetindo algumas das políticas de promoção automática nos anos 1980) na disciplina Cálculo I, campeã absoluta de reprovações. Ao invés de buscar formas adequadas para o ensino de disciplinas abstratas e complexas (Crato, 2006), propõe-se que, nelas, não seja avaliado o aprendizado. Não importa que os alunos aprendam, e sim que obtenham os diplomas. Boa parte dos cursos de educação matemática e as etno-disciplinas diversas funcionam dessa forma: recusa-se aos alunos o direito de aprender em troca de lhes oferecer a dádiva do diploma (Barbosa, 2010; Crato, 2006).

Nesse quadro, a instituição escolar apareceria quase apenas como um mecanismo de legitimação da herança social através da diplomação dos filhos das famílias mais afluentes, até em algumas universidades de qualidade, sem qualquer papel importante na transmissão de conhecimentos ou na preparação técnica para o trabalho. Nesse sentido, os diplomas superiores teriam um valor fortemente posicional. Claro que isso é bem sabido (Bertolin, 2011; Schwartzman, 2011), e uma possível inovação da nossa análise seria associar o título posicional ao academicismo e ao patrimonialismo. Tudo somado e conduzindo a ineficiências do sistema.

\section{Limites de uma possível democratização}

Em termos de análises sociológicas mais qualificadas, aborda-se, aqui, uma questão importante e bastante paradoxal, que poderia ser enunciada da seguinte forma: mesmo com uma engenharia institucional eficiente (do ponto de vista da democratização das oportunidades educacionais), que conseguisse excluir totalmente a dimensão ritualística da credencial acadêmica no mercado de trabalho, deixando somente sua dimensão indicadora de mérito, ainda assim não poderíamos pensar num sistema democrático com igualdade de oportunidades na alocação de postos de trabalho. 
Essa maior equalização das oportunidades não ocorreria ainda assim, pois os próprios critérios meritocráticos, como já sugeria Weber (1975, p. 240-241), podem expressar uma dimensão estamental do grupo que controla a elaboração e a legitimação deles. Nesse ponto, recorremos novamente a Margaret Archer (1982): usar a educação como critério para alocar posições na sociedade é certamente mais democrático do que utilizar critérios adscritos, como a família de origem, a raça ou o sexo, tipicamente patrimoniais. Isso não muda o fato de o uso da escolaridade como critério ser expressão de uma forma específica de dominação legítima, no sentido clássico dado por Weber: trata-se da probabilidade de adesão, em alguma medida voluntária, por parte dos dominados, às regras de funcionamento do sistema de ordem social (Weber, 1964). Nesse sentido, compreende-se a valorização da escola, mesmo (em alguns casos, principalmente) por aqueles que recebem tão pouco dela. E compreende-se o desprezo de algumas aristocracias ou elites tradicionais pelas formas modernas de conhecimento científico, base do domínio das profissões tal como elas existem atualmente.

Num estudo bastante recente e que mostra como o mundo tem se transformado radicalmente pela importância dada à educa농 ção, principalmente aquela de nível terciário, : Baker (2014) argumenta que se produziu uma verdadeira revolução educacional. Essa revolução teria criado uma sociedade que, em seus of princípios de funcionamento e hierarquização, seria definida pela educação. Numa aborलै dagem que talvez se aproximasse daquela de $\stackrel{2}{+}$ Raymond Williams e sua Long Revoluton (não \& mencionada no texto, no entanto), Baker des$\stackrel{\infty}{\sim}$ taca inúmeras dimensões que se reorganiza-

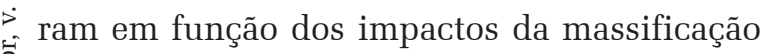
da escolaridade. O texto merece uma discussão mais detida. Entretanto, reforça o nosso argumento do domínio da escolaridade ou da educação certificada como elemento essencial das formas sociais modernas. Na medida em que o ensino superior se destaca como um dos principais recursos legítimos para a conquista e manutenção de posições sociais privilegiadas, a definição das regras do jogo social, nesse campo, torna-se um componente crucial das relações de poder.

\section{Domínio estamental, cultura profissional}

$\mathrm{O}$ argumento, assim, vai mais longe e qualifica o tipo de domínio, especialmente aquele existente no SES. Não é apenas uma valorização dos certificados escolares. É também a instalação de formas específicas de perceber e organizar o mundo, marcadas pelo profissionalismo e pelo cientificismo, tal como são definidos em cada grupo que tem controle político, econômico e social sobre o SES. Ou seja, é o domínio do certificado escolar com características estamentais dos grupos profissionais que controlam o SES. Assim, a perspectiva médica organiza a área de saúde, tanto nas formas da divisão técnica do trabalho entre as diferentes especialidades médicas quanto nas hierarquias entre os médicos e entre eles e os demais profissionais da área de saúde. O mesmo acontece com as áreas do direito ou da engenharia. Considerando-se apenas as profissões imperiais (Coelho, 1999; Vargas, 2010), já se tem vasto material para entender o significado desses traços estamentais e seu impacto sobre o SES. Este último se torna um campo (no sentido bourdieusiano do termo) onde se travam verdadeiras batalhas sobre qual deve ser o conteúdo das formações diversas, quais as relações entre elas, quais as prioridades e os ordenamentos. Tomando como exemplo a preparação profissional dos professores, fica evidente o impacto das regras sociais e organizacionais do SES. Como demonstrou Schwartzman (2003), a formação de professores sempre foi relegada às partes menos prestigiadas desse sistema. Atualmente, a mesma formação é feita nos cursos noturnos de licenciatura nas universidades públicas ou nas licenciaturas em instituições particulares. Todos eles, ou quase, são destinados aos alunos 
que são os primeiros de suas famílias a ter acesso ao ensino superior. ${ }^{4}$

Numa análise que foi objeto de debates importantes, Garry Stevens (1991) assumia claramente para a arquitetura, sua área de atuação, os sentidos dessa atuação corporativa.

The basic idea is that architecture reproduces itself through a formal system of education, which is properly located in universities. The state credentials graduates, formally certifying them as competent, relying on professional proxies to monitor the quality of educational programs. Apart from teaching, the academics also produce research or scholarship, which informs their teaching and increases the knowledge base of the profession. ${ }^{5}$

Isso tudo indica que todo o sistema, ainda que revestido de todos os parâmetros de igualdade e legitimidade, continua sendo afetado, em seu funcionamento, por critérios alheios ao sistema meritocrático, como bem tem demonstrado a literatura sociológica contemporânea sobre o nível de desigualdade do acesso aos sistemas de ensino superior, devido à fatores relacionados à origem social das pessoas. (Hout; Raftery; Bell, 2003; Lucas, 2001. Prates; Collares, 2015). São produtos sociais de disputas estamentais. Mais democráticas, talvez, mas certamente não neutras. Nesse sentido, pode-se sugerir, com base na sociologia das profissões, que, mesmo considerando o diploma como credencial acadêmico-meritocrática, o controle social exercido pelo estamento profissional sobre os tipos de conhecimento por ele requerido o transformaria em recurso de poder social. No contexto brasileiro atual, esse fato poderia ser visto como um possível retrocesso histórico pela revitalização, com roupagem nova, de um sistema credencialista estamental típico do modelo da "velha" sociedade patrimonial.

\footnotetext{
${ }^{4}$ Os estudos incluídos no livro organizado por Heringer e Honorato (no prelo) dão excelentes indicações sobre o modo de funcionamento e o público destinado aos cursos de Pedagogia.

5 Disponível em <http://www.archsoc.com/kcas/Historyed.html >. Acessado em 15/01/2015.
}

\section{As desigualdades sociais a partir do SES}

A força dos títulos acadêmicos, ou do viés acadêmico, parece ter também um efeito de desqualificação da experiência e de outros títulos, valorizando os bacharelados em detrimento das licenciaturas e dos cursos tecnológicos, como critério principal de hierarquização social. Trata-se de uma expressão das dimensões patrimoniais que organizariam o domínio estamental e profissional moderno num sistema de ensino superior ainda marcado pelas formas tradicionais de exercício do poder. Talvez a forma mais adequada de exame dessa questão seja verificar como funcionam os fatores, tais como os diferentes tipos de diplomas do ensino superior, e o tipo de instituição que emite esses diplomas na definição das posições ou ocupações no mercado de trabalho.

A contribuição da sociologia das organizações para a análise da educação superior tem destacado o peso dos fatores "tipo de diploma”, por um lado (se mais acadêmico ou profissional), e tipo de instituição educacional que emite o diploma, por outro (se mais profissional ou mais acadêmica). Prates (2007) tem insistido que o ambiente institucional das organizações educacionais que emitem os diplomas pesa muito na determinação do "valor" do diploma. No Brasil, por exemplo, as instituições públicas oferecem um clima acadêmico, como ambiente de aprendizagem, que, independentemente do conteúdo programático do curso, aumenta diferencialmente as habilidades profissionais dos seus alunos em relação às suas congêneres privadas. O estudo de Prates e Faleiros (2014), com dados da Pesquisa Nacional por Amostra por de Domicílios (Pnad), mostra, por exemplo, que os egressos de cursos tecnológicos das instituições públicas têm uma chance maior do que os formados em instituições privadas de assumirem postos de trabalho de maior prestígio ocupacional. Mas, por outro lado, como sugere Barbosa (2010a), no Brasil, hoje, a credencial vocacional do tecnólogo é socialmente desva- 
lorizada frente à do bacharel. Há, sim, um peso importante, em termos de prestígio social, do diploma de bacharelado de quatro anos relativamente ao de curso tecnológico de dois anos, ou ate mais longo, independentemente do diferencial de conhecimento que cada um possa oferecer. É importante notar que os saberes práticos, dominantes nos cursos de natureza vocacional ou tecnológica, ${ }^{6}$ são extremamente valorizados em empresas e mesmo pelos trabalhadores. No entanto, as regras em torno das credenciais e do seu uso no sistema produtivo dão ênfase ao título de bacharel (Barbosa, 2010). No estudo de Prates e Faleiros, mencionado acima, quando é analisado o caso da Universidade de São Paulo - Leste (USP-LES$\mathrm{TE}$ ), percebe-se claramente que, embora todos os cursos oferecidos nessa instituição fossem de bacharelado com duração de quatro anos, o conteúdo desses cursos era tipicamente de natureza vocacional, voltado para preparação de trabalhos específicos. Os próprios professores sofriam as consequências emocionais de certa dissonância cognitiva: eles eram, em sua grande maioria, jovens doutores, formados em universidades de prestígio acadêmico e, embora estivessem na USP, a melhor universidade brasileira, trabalhavam em uma escola "quase vocacional". Durante todo tempo da entrevista, 늘 esses profissionais faziam questão de enfatizar $\stackrel{\sim}{\circ}$ que a missão deles, na instituição, era não deixá-la perder o vínculo com a pesquisa básica, herdado da "instituição-mãe”, a USP-Butantã.

\section{CONCLUSÕES}

i.

ז

O bacharelismo academicista constitui um fator determinante no sistema de apropria-

${ }^{6}$ As definições de educação vocacional são muito variadas. No entanto, no texto mencionado acima, Wolf (2001, స్ p. 58) oferece uma noção razoável. " 'Vocational education' instead refers to courses for Young people which are of-

II fered as a lower-prestige alternative to academic second-

ory schooling, and which lead to manual, craft, and, more

$\supsetneq$ recently, secretarial jobs. 'Technical' education slots into

the hierarchy above vocational and below academic, and

leads, in theory, to the technician jobs which increased in

¿ number during the twentieth century." ção, produção e distribuição dos conhecimentos científicos e técnicos. Isso pode ser traduzido de duas formas. Primeiro, pelo efeito indireto do aumento de capital social que esses cursos oferecem, especialmente se associados ao formato institucional de uma instituição de pesquisa. Segundo, pelo efeito simbólico de status que os associa a uma característica relevante do estilo de vida das elites patrimoniais. De outra parte, entretanto, o bacharelismo está no cerne do dilema sugerido, de acordo com Weber, pelo processo de racionalização na sociedade ocidental. Nesse caso, caberia perguntar se não seria o credencialismo uma resposta ótima à demanda do sistema meritocrático requerido pelo processo, generalizado na sociedade, de burocratização racional-legal. ${ }^{7}$

Mas então, sendo assim, mesmo que o diploma traga, como credencial, um conteúdo técnico, esse conteúdo de conhecimento pode estar refletindo as demandas estamentais de fechamento de acesso, e não as necessidades reais do processo tecnológico em questão. Questão essencial para uma boa sociologia dos currículos, ela demandaria estudos que saíssem das generalidades sobre o funcionamento de escolas subordinadas ao capitalismo para chegar às sutilezas e especificidades das diferentes formas de conhecimento (e ensino) em cada área.

As dificuldades do sistema de ensino superior em acompanhar a evolução da divisão técnica dos trabalhos, tornando-se incapaz de qualificar adequadamente para o mercado, poderiam estar associadas ao domínio dessa perspectiva, que, também, se ligaria às ineficiências na produção de inovações e no registro de patentes, já devidamente destacadas nos estudos de Guimarães (2011).

Nesse quadro, a ineficiência social seria mais destacada, diante do alargamento das políticas de inclusão no sistema universitário: os

\footnotetext{
${ }^{7}$ Obviamente, quando se fala aqui em burocracia e sistema educacional, não estamos assumindo que essas sejam "forças" estruturais objetivas que moldam o comportamento das nossas instituições. Nesse ponto, concordamos totalmente com Collins quando enfatiza o conflito de interesses e de ideologias como fonte primordial de colocação em
} marcha dessas "forças" estruturais. 
novos grupos que conseguem entrar para um curso superior seriam excluídos (do interior, como diria Pierre Bourdieu,), seja pela desvalorização de alguns títulos, seja pela subordinação de carreiras e de tipos de formação.

A ineficiência social apareceria como o resultado mais central do academicismo tal como ele se organiza no SES brasileiro. O Academicismo apareceria como um retrocesso da forma de dominação, reafirmando o bacharelismo ou patrimonialismo contra uma perspectiva mais técnica ou mais profissional.

Embora analisando de uma perspectiva distinta, o texto de Dubet, neste dossiê, destrincha as várias dimensões imbricadas no fenômeno da democratização do sistema de ensino superior nas sociedades contemporâneas, enquanto o nosso trata da questão do uso dos diplomas como marca social de status, favorecendo os grupos sociais que podem monopolizar o acesso a eles. Contudo, de uma maneira geral, essa perspectiva pode ser incluída na mesma temática da relação entre a credencial do diploma escolar e os seus efeitos sobre a mobilidade social através do mercado de trabalho. Coloca-se em pauta uma questão. Poderia o viés acadêmico, ou o domínio de um bacharelismo credencialista, reduzir ou mesmo anular a força ou o espaço do conhecimento ao dar toda a força ao social, ao título e não ao saber? Poderia ele reduzir o impacto potencialmente democratizante da expansão tão significativa do nosso sistema de ensino superior?

Se tais hipóteses se confirmam, estamos diante do quadro que começou a ser esboçado por Goldthorpe (1997), quando o autor inglês aponta as dificuldades para o funcionamento de uma sociedade minimamente meritocrática. Entre essas dificuldades, destacam-se as estratégias dos grupos de elite que se valem do sistema escolar para ocupar as posições superiores da hierarquia social. O alongamento dos estudos para além do suportável por famílias mais pobres é uma dessas estratégias. Talvez a diversificação diferenciada e desigual do sistema de ensino superior brasileiro possa ser considerada, como já indicava Ferreira (2000), uma expressão do domínio das classes médias educadas sobre o sistema de ensino como um todo. Não se trata de ações conscientes, claramente voltadas para a exclusão desses novos grupos que chegam ao ensino superior. Seria apenas a expressão das formas de perceber o mundo que estabelecem aquele problema clássico da vida social: a perfeita racionalidade das escolhas individuais pode se combinar em efeitos perversos coletivos, não desejados por nenhum desses atores ou agentes. No caso, a geração de um sistema de ensino superior que é excludente, mesmo quando abre suas portas para grupos sociais antes ausentes. É quase como se esse sistema abrisse, ao lado da porta principal, uma série de entradas de serviço. Que só dão acesso aos espaços subordinados.

Recebido para publicação em 15 de janeiro de 2015 Aceito em 15 de março de 2015

\section{REFERÊNCIAS}

ADORNO, S. Os aprendizes do poder. O bacharelismo liberal na política brasileira. São Paulo, Paz e Terra, 1988, 202 p.

ARCHER, M. S. (ed.). The sociology of educational expansion: take-off, growth and inflation in educational systems. London: Sage, 1982, 314 p.

BAKER, David P. The schooled society: the educational transformation of global culture. Stanford, California: Stanford University Press, 2014, 342 p.

BARBOSA, Maria Ligia O. As profissões no Brasil e sua sociologia. Dados, Rio de Janeiro, v. 46, p. 593-607, 2003.

Diplomas e saberes: acadêmicos, tecnólogos e desigualdade no Brasil. In: FARTES, V. (org.). Educação, (trans)formação e saberes profissionais no mundo em mudanças. Salvador: EDUFBA, v.1, p. 67-101, 2010a

. Desafios da escola contemporânea: a formação do educador In: FAZZI, R. et al (orgs.). Diálogos em extensão: encontros da Rede PUC sobre Infância, Adolescência e Juventude. Belo Horizonte: Editora PUC Minas, p. 135155,2010

The expansion of higher education in Brazil: credentials \& merit, REMIE - Multidisciplinary Journal of Educational Research, v. 2, n. 3, p. 251-271, October, 2012.

. O ensino superior no Brasil: credencial, mérito e os coronéis. In: (Org.). Ensino Superior: expansão e democratização. Rio de Janeiro: 7Letras, p. 51-69, 2014.

; ZUCARELLI, Carolina: A formação nas áreas de ciência, tecnologia, engenharias e matemática (CTEM) em nível superior no Brasil: saberes modernos em instituições tradicionais. In OLIVEIRA, Marina et al (Orgs.) Rede de Pesquisa Formação e Mercado de Trabalho, Coletânea de artigos, v. 4. Formação de Profissionais das áreas de 
Ciência, Tecnologia, Engenharia e Matemática, Brasília, ABDI/IPEA, 2014.

BERTOLIN, Julio C. G. Os quase mercados na educação superior: dos improváveis mercados perfeitamente competitivos à imprescindível regulação do Estado... Educação e Pesquisa, São Paulo, v. 37, n. 2, p. 237-248, mai./ago., 2011.

BILLS, D. B. The sociology of education and work. Malden, MA: Blackwell Publishers Ltd., 2004, 302 p.

BRAGA, Mauro M.; PEIXOTO, M.C.; BOGUTCHI, T. Tendências da demanda pelo ensino superior: estudo de caso da UFMG. Cadernos de Pesquisa, São Paulo: Fundação Carlos Chagas, n. 113, p. 129-152, 2001.

BRUNELLO, G.; GARIBALDI, P.; WASMER, E. Education and training in Europe. Oxford: Oxford University Press. 2007, $478 \mathrm{p}$.

CAMPANTE, Rubens G. O patrimonialismo em Faoro e Weber e a sociologia Brasileira. DADOS - Revista de Ciências Sociais, Rio de Janeiro: Instituto de Estudos Sociais e Políticos, v. 46, n. 1, p. 153 a 193, 2003.

CARVALHO, J.M. A escola de Minas de Ouro Preto: o peso da glória. Rio de Janeiro: Finep Cia Editora Nacional, 1976, 202 p.

COELHO, E. C. As profissões imperiais. Rio de Janeiro: Record, 1999, $304 \mathrm{p}$.

CRATO, Nuno. $O$ 'Eduquês em discurso directo - Uma crítica da Pedagogia Romântica e Construtivista, Lisboa: Gradiva, 2006, 132 p.

DULCI, O.S. As elite mineiras e a conciliação: a mineiridade como ideologia. Mimeo, ANPOCS, 1983.

Política e economia em Minas Gerais: um balanço dos anos 90. In: ; PAIVA, P.T.A. (Org.). 20 Anos do Seminário sobre a Economia Mineira. Belo Horizonte: UFMG/CEDEPLAR, v. 3, p. 101-112, 2002.

FERREIRA, Francisco. Os determinantes da desigualdade de renda no Brasil: luta de classes ou heterogeneidade educacional. In: HENRIQUES, Ricardo (org.) Desigualdade e pobreza no Brasil. Rio de Janeiro: IPEA, p. 131-158, 2000. Também disponível em:<http://www.econ.puc-rio.br/pdf/ td415.pdf $>$.

GOLDTHORPE, J. Problems of 'Meritocracy'. In: HALSEY; LAUDER; BROWM; WELLS (Eds.): Education: culture, economy, society. Oxford: University Press, Oxford, p. เ2 663-682, 1997.

จ GUIMARAES, Sonia K. Empreendedorismo intensivo $\therefore$ em conhecimento no Brasil. Cad. CRH [online], v. 24, $\sum$ n. 63 [citado 2015-01-16], p. 575-592, 2011. Disponível em: <http://www.scielo.br/scielo.php?script=sci 匹 arttext\&pid=S0103-49792011000300008\&lng=pt\&nrm=

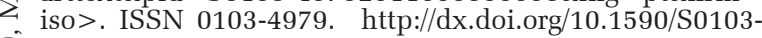
of 49792011000300008.

I GUSSO, Divonzir A.; NASCIMENTO, Paulo E. M. Contexto ले e dimensionamento da formação de pessoal técnico¿. científico e de engenheiros. Radar: tecnologia, produção ; e comércio exterior. Brasília: IPEA, n. 12, p. 23-34, 2011.

- HOUT, Michael; RAFTERY Adrian E.; BELL, Eleonor O. Making the Grade: Educational Stratification in the United $\stackrel{\infty}{\sim}$ States, 1925-1989. In: Persisting inequality: Educational $\because$ Stratification in Thirteen Countries. Editado por Yossi Shavit e Hans-Peter Blossfeld. Boulder, CO: Westview Press, p. 25-50, 2003.

$\Xi$ LUCAS, Samuel R. Effectively Maintained Inequality: क Education Transitions, Track Mobility, and Social I Background Effects. The American Journal of Sociology. n. 106 (6) p. 1642-1690, 2001

O MARTINS, Carlos B. Uma Reforma Necessária. Educação e Sociedade. Campinas: CEDES/UNICAMP, v. 27, n. 96 Especial, p. 1001-1020, out. 2006.
MENDONÇA, Erasto Fortes: A regra e o jogo: democracia e patrimonialismo na educação brasileira. Tese de doutorado, Faculdade de Educação, Unicamp, 2000.

NASCIMENTO, P. A. M. M. et al. A questão da disponibilidade de engenheiros no Brasil nos anos 2000. Radar: tecnologia, produção e comércio exterior. Brasília: Ipea, n. 32, abr. 2014.

OJALA, Raisa M. P. Projeto de futuro de jovens universitários no distrito federal: um estudo de caso. Doutorado em Sociologia, Universidade de Brasília, 2008, 307 p.

PAUL, J. J.; VALLE-SILVA, N. do. Conhecendo o seu lugar: a auto-seleção na escolha da carreira. Revista Brasileira de Política e Administração da Educação. Goiás: Associação Nacional de Política e Administração em Educação. n. 14 (14), p. 115-130, 1998.

PRATES, A. A. P. Universidades VS Terceirização do ensino superior: a lógica da expansão do acesso com manutenção da desigualdade. O caso brasileiro. Sociologias v. 9, n. 17, p. 102-123, Jan./jun., 2007.

; FALEIROS, M. S. Os efeitos diferenciais do tipo de instituição de ensino superior sobre o prestígio dos seus egressos no mercado de trabalho. In: BARBOSA, M. de O. (Org.) Ensino superior: expansão e democratização. Rio de Janeiro: 7letras, p.129-154, 2014.

; COLLARES, A. C. M. Desigualdade e expansão do ensino superior na sociedade contemporânea: o caso brasileiro no fina do Século XX e princípio do Século XXI. Belo Horizonte: Fino Traço, 2015 (no prelo).

SCHWARTZMAN, S A força do novo: por uma sociologia dos conhecimentos modernos no Brasil. Revista Brasileira de Ciências Sociais. v. 2, n. 5, out. 1987.

The Challenges of Education in Brazil. Visiting Fellow: Centre for Brazilian Studies, Oxford University Version 3, fev. n. 26, 2003

Disponível em: <http://www.schwartzman.org.br/simon/ pdf/challenges.pdf $>$.

Atualidade de Raymundo Faoro. DADOS - Revista de Ciências Sociais, Rio de Janeiro, v. 46, n. 2, p. 207-213, 2003.

O viés acadêmico na educação brasileira. In: BACHA, Edmar; SCHWARTZMAN, S (Orgs.). Brasil: a nova agenda social. Rio de Janeiro: LTC, p. 254269, 2011. Disponível em: https://archive.org/stream/ ANovaAgendaSocial/nova_agenda\#page/n12/mode/1up.

SHAVIT, Y.; BLOSSFIELD, H-P. Persistent equality: changing educational attainment in thirteen countries. Boulder: Westview Press, 1993, 504 p.

; MILLER, W. From school to work: a comparative study on education qualifications and occupational destinations. Oxford: Claredon Press, 1998, 550 p.

STEVENS, Garry. The Social Foundation of Architectural Distinction. CAMBRIDGE: MIT Press, 1991.

VARGAS, Hustana Maria. Sem perder a majestade: 'profissões imperiais' no Brasil. Estudos de Sociologia, São Paulo, v. 15, p. 107-124, 2010.

WEBER, Max. Economia y Sociedad. WINCKELMANN, Johannes e Echavarria, José Medina (Eds.). México: Fondo de Cultura Económica, v. 1, p. 15-30, 170-172, 1964. Dinponível também em: https://zoonpolitikonmx.files.wordpress. com/2014/08/max-weber-economia-y-sociedad.pdf.

From Max Weber: essays in sociology. Gerth and Mills (eds.). New York: Oxford University Press, 1975.

WOLF, Alison: Does education matter? Myths about education and economic growth. London: Penguin books, 2001, 332 p.

ZAGO, Nadir: Do acesso à permanência no ensino superior: percursos de estudantes universitários de camadas populares Revista Brasileira de Educação, v. 11, n. 32 , p. 226-237, maio/ago. 2006 


\section{THE EXPANSION AND DEMOCRATIZATION POSSIBILITIES OF HIGHER EDUCATION IN BRAZIL}

\author{
Antonio Augusto Pereira Prates \\ Maria Ligia de Oliveira Barbosa
}

\section{L'EXPANSION ET LES POSSIBILITÉS D' DE DÉMOCRATISATION DE L'ENSEIGNEMENT SUPÉRIEUR AU BRÉSIL}

\author{
Antonio Augusto Pereira Prates \\ Maria Ligia de Oliveira Barbosa
}

Given the expansion and diversification of the recent Brazilian higher education system, this article discusses possible trends for its modernization and democratization. The article seeks to develop issues on the values socially assigned to higher education diplomas. It focuses mainly on the relationship between the almost absolute dominance of a patrimonial vision on higher education and the fraying of scientific/ or pedagogical contents associated with various diplomas. Patrimonialism appears in the preference by BA at the expense of license and technological diplomas; in vocations socially marked; in the schedules and shifts of different courses; in the lack of interest on technical areas and in the pedagogy of cultivation. Moreover, the patrimonial view appears in the Dialectic of Knowledge and the Social Force of diplomas. The possible installation of a radical credentialism in Brazil allows for the analysis of the relationship between patrimonial dominance and the professional culture, indicating the limits of the higher education system democratization.

KEY wORDS: Higher Education. Credentials. Professional culture. Patrimonialism.
Compte tenu de l'expansion et la diversification récentes du système d'enseignement supérieur au Brésil, cet article examine les tendances possibles pour la modernisation et la démocratisation de ce système. L'article cherche à développer des questions associées aux valeurs socialement attribuées à des diplômes d'enseignement supérieur. Il analyse principalement la relation entre la domination d'une vision patrimoniale sur l'enseignement supérieur et l'effilochage du contenu pédagogique et/ou scientifique associé à divers diplômes. Le patrimonialisme apparaît dans la préférence pour le diplôme académique au détriment des certificats pour professeurs ou pour les techniciens; des vocations socialement marquées; les horaires de différents cours; le manque d'intérêt par les domaines techniques; et de la pédagogie de l'homme cultivé. En outre, le patrimonialisme apparaît dans la dialectique entre la connaissance et la force sociale et les diplômes. L'installation possible d'un "diplômanie" radical au Brésil permet d'analyser la relation entre la domination patrimoniale et la culture professionnelle, indiquant les limites de la démocratisation du système d'éducation supérieures.

Mots-Clés: Enseignement supérieur. Diplômes. Culture professionnelle. Domination traditionnelle. 
